\title{
Repeated isolated bilateral coronary ostial stenosis related to the syphilitic aortitis
}

\author{
Sang Min Kim, Sang Yeub Lee, Ju-Hee Lee, Jang-Whan Bae, and Kyung-Kuk Hwang
}

Regional Cardiovascular Disease Center, Division of Cardiology, Department of Internal Medicine, Chungbuk National University College of Medicine, Cheongju, Korea

Received: April 16, 2015

Revised : September 24, 2015

Accepted: July 11, 2016

\section{Correspondence to}

Sang Yeub Lee, M.D.

Regional Cardiovascular Disease

Center, Divison of Cardiology,

Department of Internal Medi-

cine, Chungbuk National Univer-

sity Hospital, 776 1(il)sunhwan-

ro, Heungdeok-gu, Cheongju

28644, Korea

Tel: +82-43-269-6307

Fax: +82-43-273-3252

E-mail: louisahj@gmail.com

\section{To the Editor,}

Cardiovascular manifestations of syph-

ilis are diverse and may include: (1) asymptomatic aortitis, (2) aortic branch vessel ostial stenosis, and (3) aortic aneurysms with related aortic regurgitation [1]. Isolated bilateral coronary ostial stenosis (ICOS) is a rare condition typically associated with ascending aortitis (e.g., syphilitic aortitis), Takayasu's arteritis, necrotizing arteritis, polyartertitis nodosa, Kawasaki disease, or familial hypercholesterolemia. These specific conditions-specifically syphilitic aoritis—should be considered as potential causes when presented with a case of ICOS involving aortic arch wall thickening in the absence of major risk factors for atherosclerotic coronary artery disease [2]. Additionally, serologic and imaging tests should be used to check for aortic-wall inflammation and an assessment of its branched vessel patency should also be performed.

A 46-year-old male recently visited our hospital because of aggravating exertional chest pain. Six months ago, and because of unstable angina, this patient received a drug-eluting stent on the right coronary artery (RCA) ostium at another hospital (Fig. 1A). At that time, the left coronary artery (LCA) was completely normal (Fig. 1B). Aspirin, clopidogrel, anti-anginal drug, and statin were prescribed following that procedure. His electrocardiogram was normal and the echocardiography showed normal left ventricular systolic function and no regional wall motion abnormality; however, the patients cardiac enzymes were mildly elevated (creatine phosphokinase 363 IU/L $[<190$ $\mathrm{IU} / \mathrm{L}]$, creatine kinase-MB $5.97 \mathrm{ng} / \mathrm{mL}$ $[<4.94 \mathrm{ng} / \mathrm{mL}$ ], and cardiac troponin $0.07[<0.1 \mathrm{ng} / \mathrm{mL}])$. His lipid profile was normal. Coronary angiography (CAG) was performed via right femoral access and he was diagnosed with unstable angina. Severe left main coronary artery (LMCA) ostial stenosis was observed with damped pressure tracing in $5 \mathrm{Fr}$ diagnostic catheter (Fig. 1C); tight stenosis of the RCA ostium was also observed (Fig. 1D). The previously implanted stent missed the RCA ostium. Upon examination of intravascular ultrasound (IVUS), severe fibro-fatty tissue growth at both coronary ostial areas was identified. A $4.5 \times 15 \mathrm{~mm}$ sized bare metal stent (PRO-Kinetic, Biotronik, Berlin, Germany) was implanted in the LMCA ostium (Fig. 2A); only balloon angioplasty was performed for RCA ostium, because we suspected radical aortic inflammatory disease as a potential cause of this recurrent ICOS.

Following the procedure, we performed specific imaging and laboratory tests to identify the cause of ICOS; laboratory tests revealed evidence of syphilis. In summary, (1) venereal disease research laboratory (VDRL) titers were 

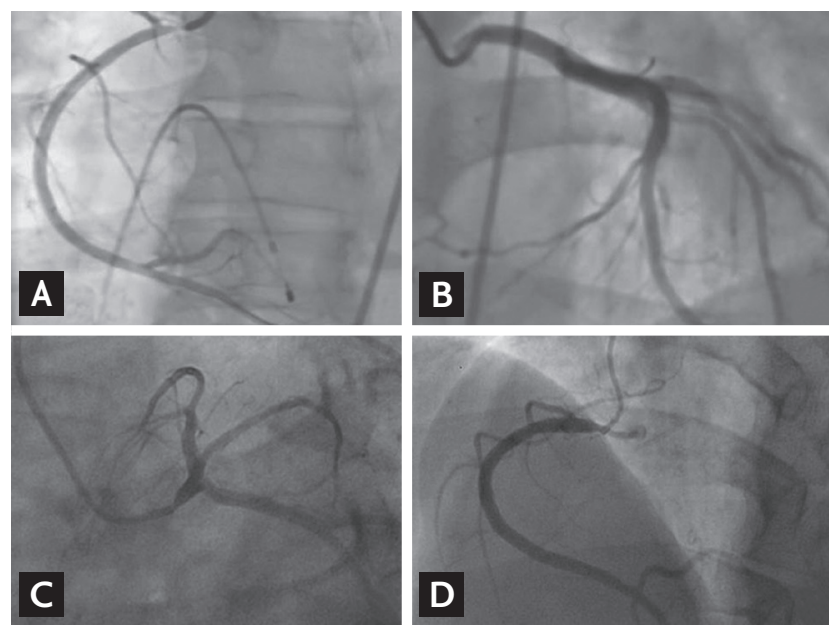

Figure 1. (A) Right coronary angiogram showing: tight stenosis of right coronary artery (RCA) ostium. Left main coronary angiogram showing: (B) a normal left main coronary artery (LMCA), (C) severe ostial stenosis of LMCA ostium. Right coronary angiogram showing: (D) restenosis of RCA ostium, (C) dilated LMCA after percutaneous coronary intervention, (D) restenosis of LMCA ostium.
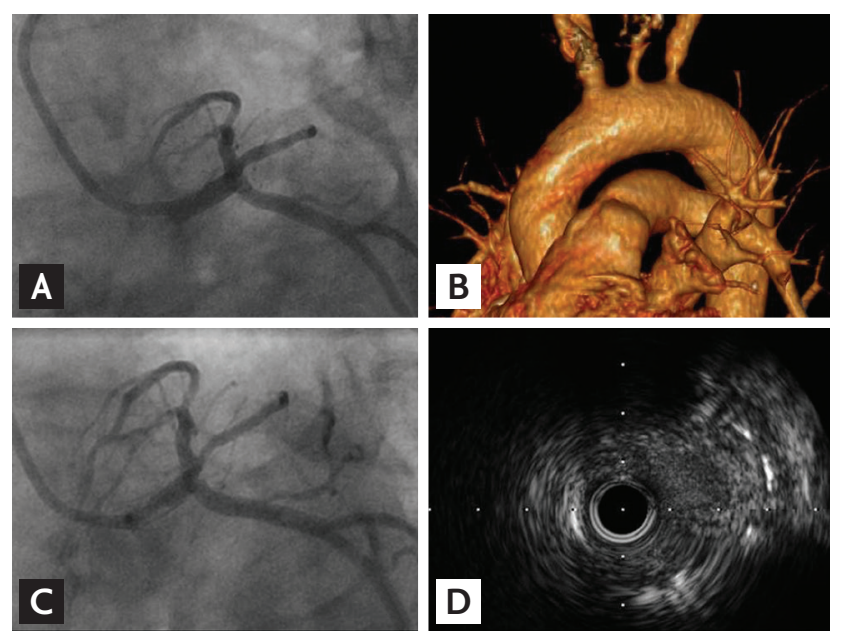

Figure 2. (A) Left main coronary angiogram showing: dilated left main coronary artery (LMCA) after percutaneous coronary intervention. (B) Thoracic computed tomography aortography: ostial stenosis of left common carotid and left subclavian artery. (C) Left main coronary angiogram showing: restenosis of LMCA ostium. (D) Intravascular ultrasound examination showing: Tissue regrowth in LMCA stenting site, especially ostium.

positive with $1 / 128$, (2) Treponema pallidum particle agglutination assay was reactive, (3) patient tested positive for fluorescent treponemal antibody absorption (FTAABS) immunoglobulin M (IgM) and FTA-ABS IgG, and (4) consecutive examinations of the cerebrospinal fluid showed neurosyphilis (120 white blood cell/high power field and reactive VDRL with 1/8 titers). Computed tomography (CT) angiography showed diffuse thickening of the thoracic aortic wall and significant ostial stenosis of both coronary arteries, the left common carotid and the left subclavian artery (Fig. 2B). We surmised that the patient suffered tertiary syphilis which involved the aortic wall, and it might have caused this recurrent ICOS. For treatment, we used high-dose penicillin G intravenous injection (6,000,000 IU/day for 14 days). Follow-up CT angiography showed patent bare metal stent on LCA ostium, and no significant difference of aortic wall thickness and stenosis of branch vessel ostium of the aorta. Positron emission tomography-CT, used to check inflammation intensity, did not identify specific abnormalities of the aorta or branched vessels 6 months after the percutaneous coronary intervention (PCI). Thirteen months after the PCI, the patient was readmitted to our hospital with aggravating exertional chest pain, and severe tissue growth was observed in LMCA ostium in CAG (Fig. 2C) and IVUS (Fig. 2D). We have performed cutting balloon angioplasty at LMCA. CT angiography showed no specific changes in aorta or the stenosis of its branched vessels. The clinical course after cutting balloon dilatation has been uneventful for 14 months.

When presented with acute coronary syndrome as outlined in the case here, emergent PCI can be a treatment option. Usual treatment modality for syphilitic aortitis-related coronary stenosis is coronary artery bypass graft [3], but coronary stenting was tried with caution recently $[4,5]$. There have been no reports about the mid or long-term clinical outcomes or prognosis in patients with syphilitic aortitis and ICOS treated with coronary intervention. This case report details a case of acute coronary syndrome caused by syphilitic coronary artery ostial stenosis treated with a drug-eluting and bare-metal stent implantation; however, recurrent episodes of restenosis were observed. If we identify another restenotic event, coronary artery bypass surgery is inevitable for this patient. After PCI for patients with ICOS, medical efforts should be given to find cause of ICOS. Especially in case of syphilitic aortitis, proper medical and image surveillance was essential to identify restenosis.

Keywords: Syphilis, cardiovascular; Coronary stenosis; Restenosis 


\section{Conflict of interest}

No potential conflict of interest relevant to this article was reported.

\section{REFERENCES}

1. Jackman JD Jr, Radolf JD. Cardiovascular syphilis. Am J Med 1989;87:425-433.

2. Machado MN, Trindade PF, Miranda RC, Maia LN. Bilateral ostial coronary lesion in cardiovascular syphilis: case report. Rev Bras Cir Cardiovasc 2008;23:129-131.
3. Tanaka K, Takeda M, Nagayama K. Composite Y-graft for syphilitic ostial stenosis in left main coronary artery. Asian Cardiovasc Thorac Ann 2007;15:159-161.

4. Kennedy JL, Barnard JJ, Prahlow JA. Syphilitic coronary artery ostial stenosis resulting in acute myocardial infarction and death. Cardiology 2006;105:25-29.

5. Nakazone MA, Machado MN, Barbosa RB, Santos MA, Maia LN. Syphilitic coronary artery ostial stenosis resulting in acute myocardial infarction treated by percutaneous coronary intervention. Case Rep Med 2010;2010:830583. 